\title{
Selective Perceptual Impairments After Perirhinal Cortex Ablation
}

\author{
Mark J. Buckley, Michael C. A. Booth, Edmund T. Rolls, and David Gaffan \\ Department of Experimental Psychology, Oxford University, Oxford, OX1 3UD United Kingdom
}

It has been suggested that the primate perirhinal cortex contributes exclusively to memory. However, recent studies in macaque monkeys have implied that the perirhinal cortex may also contribute to object perception. To investigate whether the perirhinal cortex does contribute to perception, we devised several perceptual oddity tasks in which monkeys had to choose which stimulus of several presented concurrently on a touch screen was different. Macaques with bilateral perirhinal cortex ablations were selectively impaired relative to controls at perceptually discriminating the odd stimulus when the odd stimulus was a different object and when the discrimination could not be done on the basis of simple differences in features between the stimuli. They remained unimpaired relative to controls on discriminating the odd stimulus when the odd stimulus was a different color, a different shape, or a different size even when these discriminations were extremely difficult. They were also impaired on human and monkey face oddity tasks and oddity tasks with scenes containing objects. Therefore, we reject the notion that the macaque perirhinal cortex has a role exclusive to memory and conclude that the macaque perirhinal cortex does contribute to perception. We argue that the perirhinal cortex is neither specialized for perception nor memory processes alone, but rather, is specialized for processing stimuli that require processing at a more abstract level such as at the level of an object and that the perirhinal cortex contributes to both memory and perception of such stimuli.

Key words: macaque; monkey; primate; perirhinal cortex; learning and memory; perception; amnesia; agnosia
The macaque perirhinal cortex consists of Brodmann's areas 35 and 36 and is situated in the lateral bank of the rhinal sulcus and in the laterally adjacent cortex, although the recognized extent of the perirhinal cortex differs slightly between species and across investigators (Brodmann, 1909; Amaral et al., 1987; Insausti et al., 1987).

Being located in the anterior medial part of the inferior temporal gyrus, the perirhinal cortex is sometimes considered part of the inferior temporal cortex visual system and therefore a cortical area primarily involved in object perception rather than stimulus memory. Indeed, the most prominent inputs to the perirhinal cortex $(64 \%)$ are from the laterally adjacent unimodal visual areas TE and TEO (Suzuki and Amaral, 1994a). In addition to these prominent visual inputs, the perirhinal cortex also receives inputs from polymodal association cortices, including the parahippocampal cortex $(25 \%)$, dorsal superior temporal sulcus $(6 \%)$, orbitofrontal cortex $(2 \%)$, and cingulate cortex $(<1 \%)$, and from unimodal areas including somatosensory insular cortex $(2 \%)$ and auditory superior temporal gyrus $(<1 \%)$. Although this pattern of inputs is not consistent with a purely visual perceptual role for the perirhinal cortex, it is still consistent with the view that the perirhinal cortex might have a higher-order polymodal role in object perception, associating together perceptual information about objects arising from different stimulus modalities.

However, Buckley et al. (1997) showed that perirhinal cortex lesions impaired object recognition memory but not color discrimination, whereas middle temporal gyrus lesions within visual

\footnotetext{
Received May 29, 2001; revised Aug. 27, 2001; accepted Sept. 7, 2001.

This research was supported by research grants from the Medical Research Council UK (including Medical Research Council Interdisciplinary Research Centre for Cognitive Neuroscience) and a Wellcome Trust UK studentship awarded to Michael Booth.

Correspondence should be addressed to Dr. Mark J. Buckley, Department of Experimental Psychology, Oxford University, South Parks Road, Oxford, OX1 3UD, UK. E-mail: buckley@psy.ox.ac.uk.

Copyright (C) 2001 Society for Neuroscience $\quad 0270-6474 / 01 / 219824-13 \$ 15.00 / 0$
}

area TE produced the opposite pattern of results. This functional double-dissociation suggests instead that the perirhinal cortex is functionally distinct from TE and not simply a continuation of the inferior temporal cortex (IT) perceptual system.

The perirhinal cortex is also characterized by robust interconnections with limbic system structures thought to be crucial for memory. There are strong interconnections with the hippocampal formation via the entorhinal cortex. Approximately $40 \%$ of the direct input to the anterior and lateral entorhinal cortex is provided by the perirhinal cortex, and there are also strong return projections from these same regions back to the perirhinal cortex (Insausti et al., 1987; Suzuki and Amaral, 1994b; Suzuki, 1996). Accordingly, another prevailing view is that the perirhinal cortex is involved exclusively in memory and is part of a temporal lobe limbic memory system (Buffalo et al., 1998, 1999, 2000).

However, Gaffan (1994) showed that the role of the perirhinal cortex can be doubly dissociated from that of the fornix and dissociated from the amygdala. Thus, the perirhinal cortex is functionally distinct from these limbic system structures too.

Recently, Buckley and Gaffan (1998) showed that perirhinal cortex lesions further impaired object discrimination learning tasks when object identification demands increased but memory demands remain unchanged. This implied that the role of the perirhinal cortex extends to stimulus identification and is not restricted to stimulus memory. The first aim of this study was to verify that perirhinal cortex lesions impair perception. Because a previous study (Buckley et al., 1997) found that monkeys were impaired on a color discrimination task, we also tested the hypothesis that any perceptual impairment after perirhinal cortex ablation might be specific to object perception. We developed a series of simultaneous visual discrimination ("oddity") tasks that required monkeys to discriminate between different types of stimuli. In each task the solutions were available perceptually but not from memory. We found clear support for our hypothesis that 
perirhinal lesions impair making perceptual discriminations between stimuli when the discrimination requires processing of the stimuli to an object level but not discriminations that can be done on the basis of discriminating between simple or moderately complex features of objects. We conclude that the role of the perirhinal cortex in the monkey does extend to object perception and is not restricted to object memory.

\section{MATERIALS AND METHODS}

\section{Subjects}

Eight experimentally naive juvenile rhesus monkeys (Macaca mulatta) took part in this experiment (seven females and one male). Their weights at the start of behavioral testing ranged from 2.2 to $3.7 \mathrm{~kg}$. They were housed either individually or in pairs, in rooms with automatically regulated lighting, and they were given water ad libitum. After preoperative training the animals were assigned into two groups on the basis of preoperative learning scores. Three animals received bilateral perirhinal cortex ablation (PRh), and the remaining five animals remained unoperated controls $(\mathrm{CON})$.

\section{Surgery}

The operations were performed in sterile conditions with the aid of an operating microscope, and the monkeys were anesthetized throughout surgery with barbiturate (5\% thiopentone sodium solution) administered through an intravenous cannula. A detailed description of the surgical procedure has been published elsewhere (Buckley and Gaffan, 1998c). The intended extent of the ablation included cortex in the entire rostrocaudal extent of the lateral bank of the rhinal sulcus and in the laterally adjacent $2 \mathrm{~mm}$ of cortex.

\section{Histology}

After the conclusion of all behavioral experiments, the animals with ablations were sedated, deeply anesthetized, and then perfused through the heart with saline solution $(0.9 \%)$, which was followed by formol saline solution ( $10 \%$ formalin in $0.9 \%$ saline solution). The brains were blocked in the coronal stereotaxic plane posterior to the lunate sulcus, removed from the skull, allowed to sink in sucrose formalin solution (30\% sucrose, $10 \%$ formalin), and sectioned coronally at $50 \mu \mathrm{m}$ on a freezing microtome. Every 10th section through the temporal lobe was stained with cresyl violet and mounted. The intended and actual extents of the lesions are illustrated in Figures 1 and 2. The extents of the perirhinal lesions in two subjects (PRh-2 and PRh-3) were essentially as intended with only slight encroachment into laterally adjacent cortical area TE in one hemisphere of PRh-3. The extent of the intended lesion in one subject (PRh-1) was less than intended but within the area of the intended lesion. This lesion in PRh-3 spared some cortex bilaterally in the lateral bank of the rhinal sulcus and in the cortex immediately laterally adjacent on the anterior ventral surface of the temporal lobe; this lesion also did not extend as far as intended posteriorly in one hemisphere. Thus, one subject (PRh3) acquired a subtotal perirhinal cortex lesion. In none of the subjects did the actual lesion encroach bilaterally into area TE.

\section{Apparatus}

The present tasks were performed on an automated test apparatus. The subject sat in a wheeled transport cage fixed in position in front of a touch-sensitive screen $(380 \mathrm{~mm} \times 280 \mathrm{~mm})$ on which the stimuli could be displayed. The subject could reach out between the horizontal or vertical bars (spaced $\sim 45 \mathrm{~mm}$ apart) at the front of the transport cage to touch the screen. An automated pellet delivery system, controlled by the computer, delivered reward pellets into a food well $(\sim 80 \mathrm{~mm}$ in diameter) that was positioned in front of and to the right of the subject. Banana-flavored reward pellets (190 mg supplied by Noyes Company Inc.) were only delivered in responses to a correct choice made by the subject to the touch screen. Pellet delivery was accompanied by an audible click. An automated lunch box (approximate dimensions were: length $200 \mathrm{~mm}$, width $100 \mathrm{~mm}$, height $100 \mathrm{~mm}$ ) was positioned in front of and to the left of the subject. The lunch box was spring-loaded and opened immediately with a loud crack on completion of the whole task. The lunch box contained the subject's daily diet of wet monkey chow, proprietary primate pellets, nuts, raisins, and a slice of apple, banana, and orange. An infrared camera was positioned looking down into the transport cage from above to allow the subject to be observed while it was engaged in the task. The whole apparatus was housed in an experimental cubicle that was dark apart from the background illumination from the touch screen. The presentation of the visual stimuli on the touch screen was controlled by a computer. The computer also recorded the responses that subjects made to the touch screen and controlled the delivery of reward pellets after correct responses, and it controlled the opening of the lunch box after completion of the session.

\section{Stimuli}

Each task in this study used different stimuli, which are described in detail below. In each task the resolution of the SVGA display was set at $800 \times 600$ pixels.

Oddity pretraining stimuli. The stimuli for the oddity pretraining stages were multicolored clip art images of patterns and cartoon-like drawings of objects each with dimensions of $128 \times 128$ pixels. Five sets of 10 clip art images were used for the "oddity pretraining" stages. In each trial six stimuli were displayed concurrently on the touch screen on a black background in an array consisting of two rows of three stimuli. In each trial, five of the stimuli were identical clip art images, and one stimulus was different. Figure 3(Pre 1 and Pre 2) illustrates two sample trials from the oddity pretraining stage.

Task $A$ stimuli. The stimuli for task A ("image-oddity" and "objectoddity") were digitized images of real objects presented on a touch screen. Two sets of 10 indestructible objects were used in task A. Five different views of each object were captured using a digital camera and converted to grayscale (256 levels) bitmap (BMP) files of dimensions $128 \times 128$ pixels before presentation on the touch screen. In each trial six stimuli were presented concurrently on the touch screen on a gray background. The stimulus positions were arranged in an array consisting of two rows of three stimuli with the positions of each stimulus randomized between these six stimulus positions. In the image oddity stages of task A, five of the six stimuli were identical views of one object (the particular view was chosen at random), whereas one stimulus was one
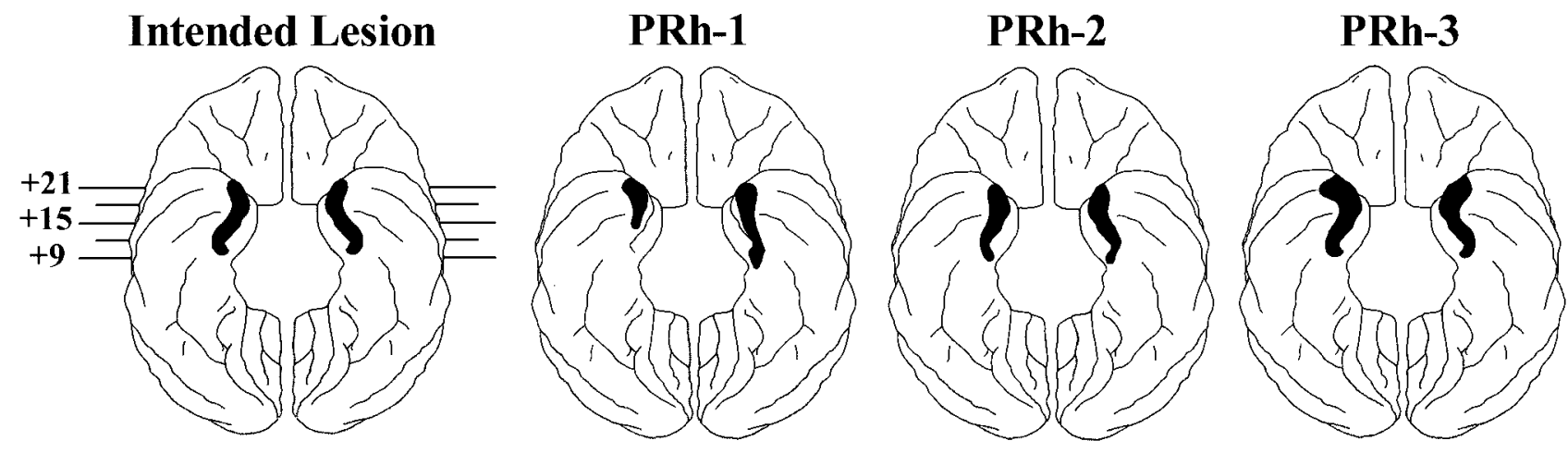

Figure 1. Drawings of ventral views of the macaque brain. The intended location and extent of the ablation of the perirhinal cortex is illustrated by the shaded region on the drawing on the left. The shaded regions on the other three drawings illustrate the actual extents of the ablations in subjects PRh-1, PRh-2, and PRh-3. To aid in visual matching of these ventral views to the coronal sections in Figure 2, the left hemisphere is shown on the right of each of these figures, and the numerals represent the distance in millimeters from the interaural plane. 


\section{Intended Lesion}
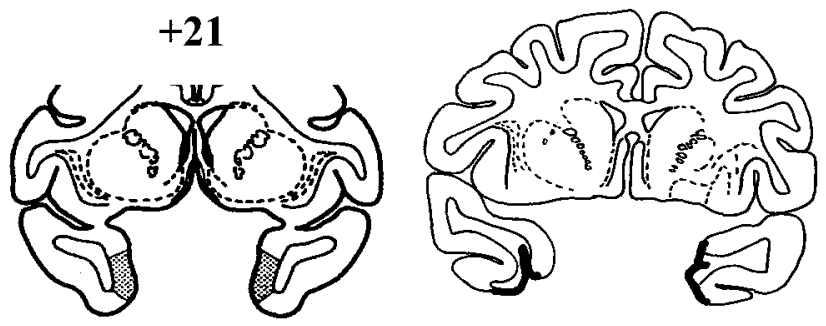

$+18$
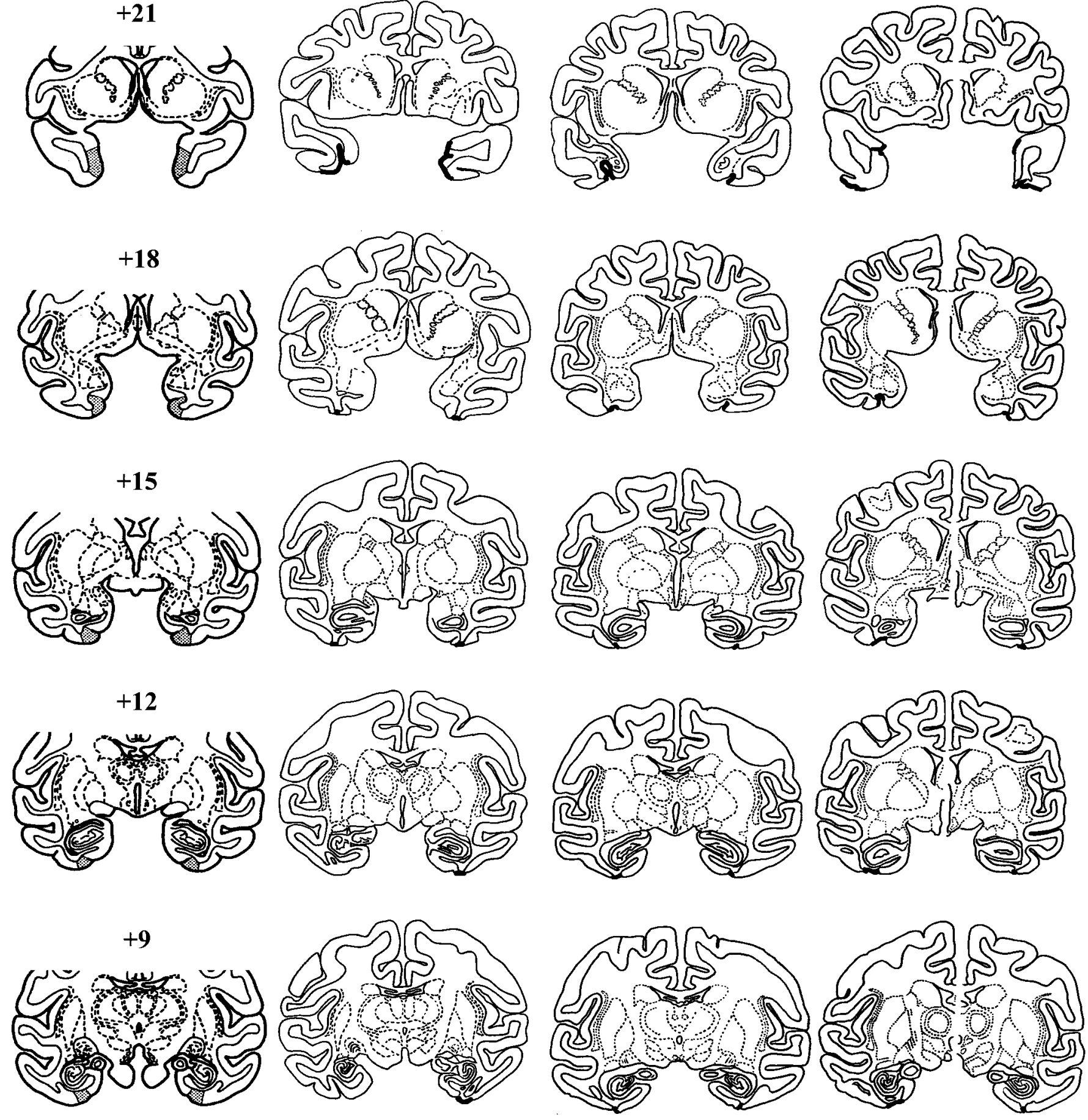

Figure 2. Drawings of coronal sections through the area of the lesion. The column on the left (Intended Lesion) shows the intended location and extent of the lesion on drawings of coronal sections from a standard macaque monkey brain from the Laboratory of Neuropsychology (National Institutes of Health, Bethesda, MD). The other three columns (PRh-1, PRh-2, PRh-3) show drawings of actual coronal sections through our lesioned monkey's brains matching those in the Intended Lesion column. Different sections were required to match the levels for the left and right hemisphere for monkey PRh-3. To aid in visual matching of these coronal sections to the ventral views in Figure 1, the left hemisphere is shown on the right of each of these figures, and the numerals represent the distance in millimeters from the interaural plane.

view of a different object from the same set (the particular view was chosen at random). Figure $3, A 1$ and $A 2$, illustrates sample trials from the image oddity stage of task A. In the object oddity stages of task A, five of the six stimuli were different views of one object, whereas one stimulus was one view of a different object from the same set (the particular view was chosen at random). Figure $3, C 1$ and $C 2$, illustrates sample trials from the object oddity stage of task A.

Task B stimuli. The stimuli for task B ("color oddity") were colored squares of dimensions $128 \times 128$ pixels. In each trial six colored squares were presented together on the touch screen on a black background and 
arranged in an array consisting of two rows of three stimuli. Nine colors were used in task B. One color designated as the base color and used in every trial together with one other color selected pseudorandomly. In each trial either five squares were the base color and one square was the other color or one square was the base color and five squares were the other color. The positions of each of the six stimuli were randomized between the six stimulus positions. Figure $3, B 1$ and $B 2$, illustrates two sample trials from task B (color oddity). The base color was an equal mix of red and green, with the red and green gun values being set at 40 , and the blue gun values being set at zero. Four of the other colors increased in relative "greenness" compared with the base color, whereas the other four colors increased in relative "redness." To generate the four colors increasing in relative greenness, we predetermined that the desired values of the green gun should be $0,37,38$, and 39 , respectively. To generate the four colors increasing in relative redness, we predetermined that the desired values of the red gun should be $0,27,34$, and 37 respectively. Then the appropriate amount of the nonspecified color (red or green) to be added to equate luminance was determined empirically using a flicker fusion technique with human subjects. There is physiological (Lee, 1991) and behavioral evidence (DeValois et al., 1974) that the visual systems of macaques and humans are very similar with respect to spectral sensitivity and flicker perception. A similar flicker fusion technique was used to generate isoluminant colored stimuli in a previous behavioral study investigating color discrimination in macaques (Buckley et al., 1997).

Task $C$ stimuli. The stimuli for task C ("shape oddity") were greencolored polygons. All of the polygons had an equal surface area but the number of sides ranged from 3 to 10 . In each trial, six polygons were presented together on the touch screen on a black background arranged in an array of two rows of three stimuli. Five polygons had the same number of sides, and one polygon had a different number of sides. The angle of rotation of each polygon around a central axis was varied randomly and independently. The positions of each of the six stimuli were randomized between the six stimulus positions. The two differentshaped polygons in each trial had either an odd or even number of sides so that the judgment of differences between the polygons could not be made purely on the basis of differences between the presence or absence of parallel sides. In any trial the discrimination could be between threesided versus five-sided polygons, four-sided versus six-sided polygons, five-sided versus seven-sided polygons, six-sided versus eight-sided polygons, seven-sided versus nine-sided sided polygons, or eight-sided versus 10 -sided polygons. The one polygon with a different number of sides could either have more or less sides than the five identical polygons. Figure 3, $C 1$ and $C 2$, illustrates two sample trials from task $\mathrm{C}$ (shape oddity).

Task D stimuli. The stimuli for task D ("degraded object oddity") were the set of digitized images of real objects used in task D (object oddity) presented in the same manner and format but obscured by a mask to degrade the view of the objects. Six different levels of masks could be applied over the digitized images to increasingly obscure the view of the digitized images. For each level of mask a fixed proportion of pixels on the screen (including pixels which were part of the objects as well as pixels that were part of the background) were switched to a random level out of 256 levels of gray. The proportions of pixels switched in this way were either $45,50,55,60,65$, or $70 \%$. Figure $3, D 1$ and $D 2$, illustrates two sample trials from task D (degraded object oddity).

Task E stimuli. The stimuli for task E ("human face oddity") were digitized images of human faces presented on the touch screen. For each of 10 different individuals the different views captured of each face were as follows: face on (looking directly ahead), left and right half profiles ( $45^{\circ}$ rotated to left or right), face on looking up (with head tilted back), and raised left and right profiles $\left(45^{\circ}\right.$ rotated to left or right looking up with head tilted back). These images were converted to $128 \times 128$ pixel grayscale (256 levels) BMP files before presentation on the touch screen. In each trial six of these stimuli were presented together on the touch screen on a gray background. The stimulus positions were arranged in an array consisting of two rows of three stimuli, and the positions of each of the six stimuli were randomized between the six stimulus positions on the screen. In the "face image oddity" stages of task E, five of the six stimuli were identical views of one face (the particular view was chosen at random), whereas one stimulus was one view of a different face (the particular view was chosen at random). Figure 3,E1 and E2, illustrates sample trials from the face image oddity stage of task E. In the face oddity stages of task E, five of the six stimuli were different views of one face, whereas one stimulus was one view of a different face with each of the six different viewing angles of faces being represented by one of the stimuli on the screen. Figure 3,E3 and E4, illustrates sample trials from the face oddity stage of task E.

Task F stimuli. The stimuli for task F (size oddity) were green outline drawings of squares presented on a black background on the touch screen. The sides of these squares were within the range of 30-128 pixels in length. For each trial two different sized squares were selected with a predetermined difference in the length of their sides (either 4, 8, 16, 32, or 64 pixels). In each trial either five larger squares were presented with one smaller square or five smaller squares were presented with one larger squares. The six squares were arranged in an array consisting of two rows of three stimuli with the positions of the six stimuli randomized between the six stimulus positions. Figure 3,F1 and $F 2$, illustrates two trials from task F (size oddity).

Task G stimuli. The stimuli for task G ("scene oddity") were digitized images of whole scenes. Each scene contained two or more different foreground objects in front of a unique background. Ten such scenes were captured using a digital camera and converted to grayscale $(256$ levels) BMP files of dimensions $368 \times 272$ pixels before presentation on the touch screen. In each trial two different scenes were chosen pseudorandomly, and then in each trial four scenes were presented together in a $2 \times 2$ array on the touch screen with three of these scenes identical and one different. The background between the scenes was black, and the positions of the four stimuli were randomized between the four stimulus positions on the screen. Figure 3, G1 and $G 2$, illustrates two trials from task $\mathrm{G}$ (scene oddity).

Task $H$ stimuli. The stimuli for task $\mathrm{H}$ ("monkey face oddity") were digitized images of monkey faces (kindly supplied by Charles Heywood) and presented on the touch screen. Three different views of five different macaque monkey faces were used. These images were in the form of a circle containing a monkey face. In each trial four of these images were presented together on a black background. The stimulus positions were arranged in an array consisting of two rows of two stimuli, and the positions of each of the four stimuli were randomized between the four stimulus positions on the screen. In the monkey face image oddity stages of task $\mathrm{H}$, three of the four stimuli were identical views of one face (the particular view was chosen at random), whereas one stimulus was one view of a different face (the particular view was chosen at random). Figure $3, H 1$ and $H 2$, illustrates two sample trials from the monkey face image oddity stage of task $\mathrm{H}$. In the monkey face oddity stages of task $\mathrm{H}$ three of the four stimuli were different views of one face, whereas the other stimulus was one view of a different face. Figure $3, \mathrm{H3}$ and $\mathrm{H} 4$, illustrates two sample trials from the monkey face oddity stage of task $\mathrm{H}$.

\section{Behavioral testing}

Summary. The subjects were tested on many different tasks during the course of this study. Therefore, a brief summary of the rationale for the series of tasks and an outline of order of testing is provided here. First the subjects learned the oddity principle (selecting the stimulus that differs) extensively preoperatively and learned to transfer the principle to new sets of stimuli. After this pretraining, the subjects were trained on image oddity and object oddity preoperatively. Although both the image oddity and object oddity tasks incorporate objects as stimuli, only the harder object oddity problems require processing at a more abstract "view-invariant" or object level. The easier object oddity problems and all of the image oddity problems can be solved purely on the basis of simple feature discrimination. To assess whether the perirhinal cortex is required for making perceptual discriminations at an object level, the $\mathrm{CON}$ and PRh groups were then retested on both of these tasks postoperatively, both with the same stimuli and with new stimuli not seen preoperatively. To assess whether any lesion effect on object oddity was specific to discriminating at an object level and not simply an impairment on any hard oddity discrimination problems, they were subsequently tested postoperatively on several other oddity tasks in the following order: color oddity, shape oddity, degraded object oddity, human face oddity, size oddity, scene oddity, and monkey face oddity. Each of these tasks is described in detail below (some of these tasks were first reported in Abstract form by Buckley et al. (1998).

Preliminary training. The subjects were first accustomed to the apparatus and taught to touch patterns appearing on the touch screen for food reward as described in detail previously (Gaffan et al., 1984).

Oddity pretraining. After completion of preliminary training the subjects started oddity pretraining on the next day. In the first stage of oddity pretraining, the subjects were trained on the oddity principle with clip art images described in the stimuli section above. Two sample trials from the 
Pre 1

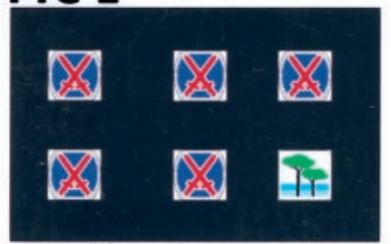

\section{Pre 2}

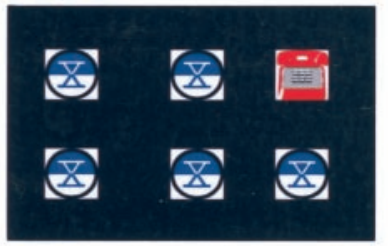

\section{C1}

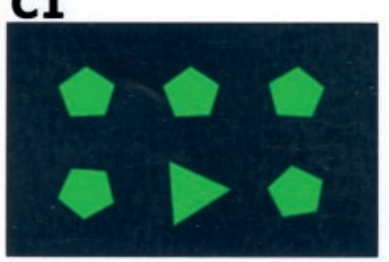

C2

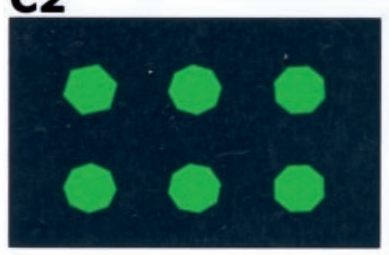

F1

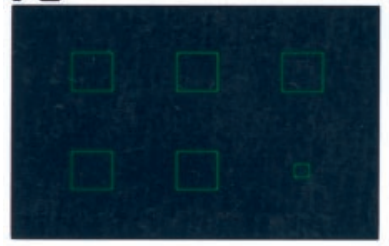

F2

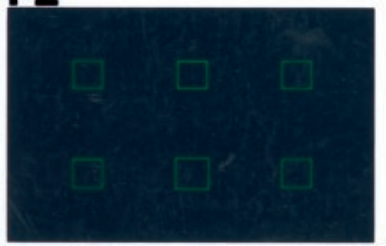

A1

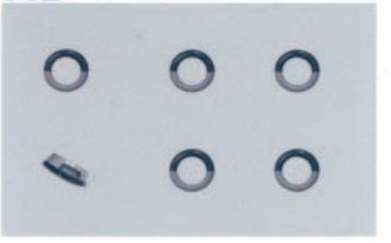

A2

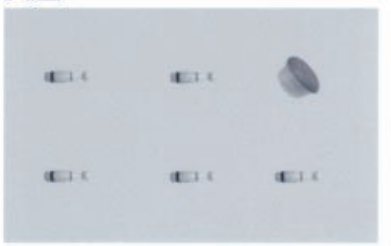

D1

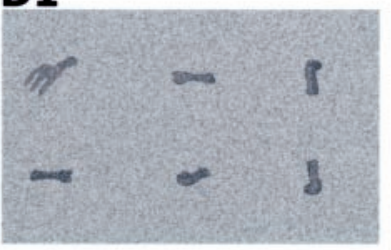

D2

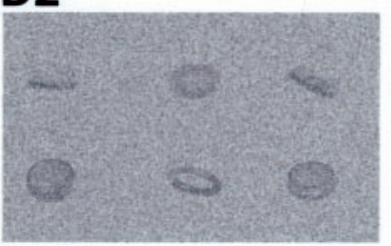

G1

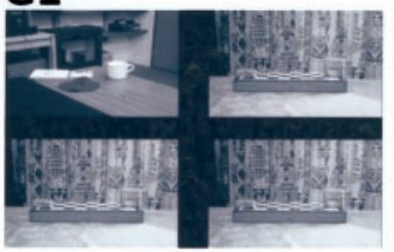

\section{G2}

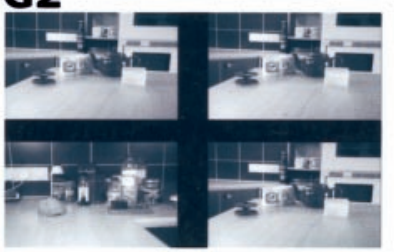

A3

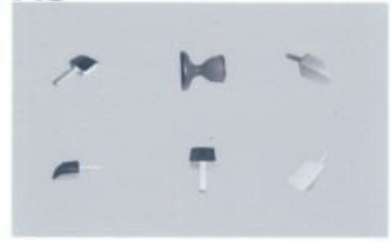

A4

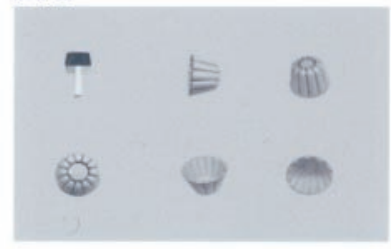

E1

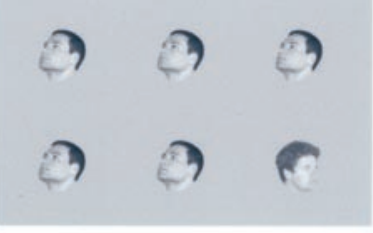

E2

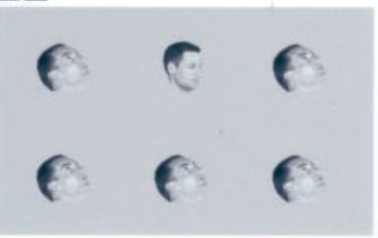

H1

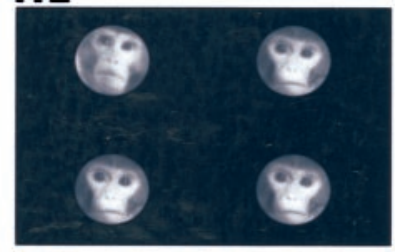

H2

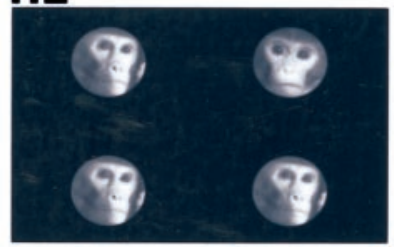

B1

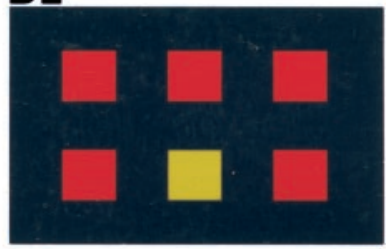

B2

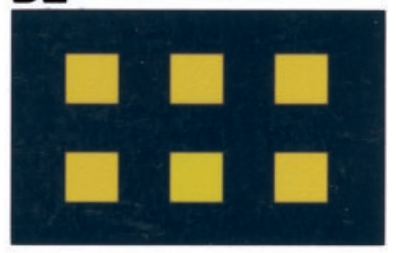

E3

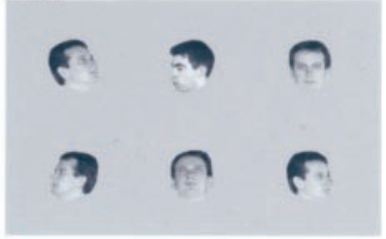

E4

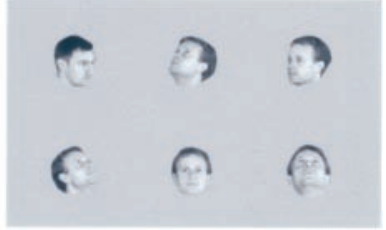

H3

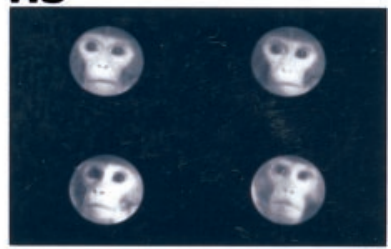

H4

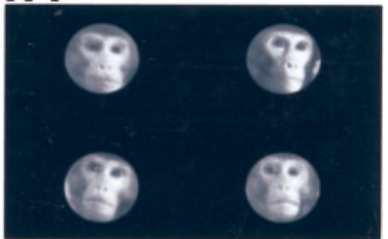

Figure 3. Pre 1, Pre 2, Two representative trials from the oddity pretraining stage, in which the subjects have to choose the odd clip art image; $A 1$, $A 2$, two representative trials from the image oddity stage of task $\mathrm{A}$ in which the subjects have to choose the odd object; $A 3, A 4$, two representative trials from the object oddity stage of task A in which the subjects have to choose the odd object; $B 1, B 2$, two representative trials, easy and hard, respectively, from task B (color oddity), in which the subjects have to choose the odd colored square; $C 1, C 2$, two representative trials, easy and hard, respectively, from task C (shape oddity), in which the subjects have to choose the odd-shaped stimulus; D1, D2, two representative trials from task D (degraded object oddity), in which the subjects have to choose the odd object behind the mask; E1, E2, two representative trials from the human face image oddity stage of task E, in which the subjects have to choose the odd individual's face; E3, E4, two representative trials from the human face oddity stage of task $\mathrm{E}$ in which the subjects have to choose the odd individual's face; $F 1, F 2$, two representative trials, easy and hard, respectively, from task F (size oddity), in which the subjects have to choose the odd-sized stimulus; $G 1, G 2$, two representative trials from task G (scene oddity), in which the subjects have to choose the odd scene; $H 1, H 2$, two representative trials from the monkey face image oddity stage of task $\mathrm{H}$, in which the subjects have to choose the odd individual's face; $\mathrm{H3}, \mathrm{H} 4$, two representative trials from the monkey face oddity stage of task $\mathrm{H}$, in which the subjects have to choose the odd individual's face.

oddity pretraining stage are illustrated in Figure 3 (Pre 1 and Pre 2). The subjects were required to touch the $\mathrm{S}+$, which was the one stimulus of the six that was the "odd-one-out", that is the different stimulus. If the subjects touched an area of the touch screen in which a stimulus was not displayed, then nothing happened, and the stimuli remained on the touch screen until one of them was touched. If the subject touched the odd stimulus $(\mathrm{S}+)$, then a reward pellet was delivered, and the rest of the stimuli disappeared from the screen apart from the odd stimulus, which 


\section{Ai}

Object Oddity
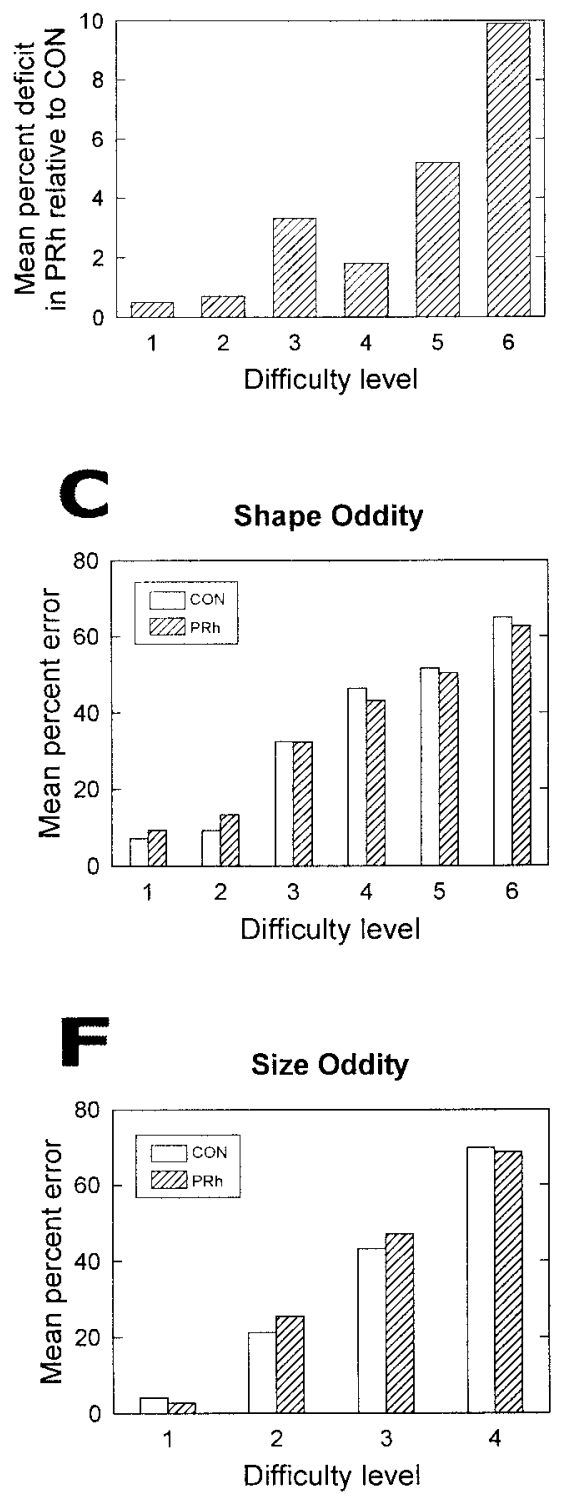

\section{Ail}

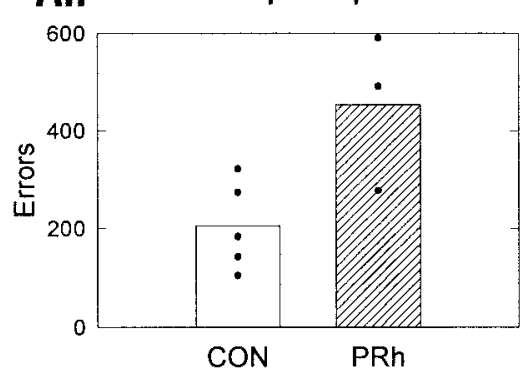
Object Oddity
with new post-op. stimuli

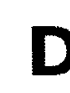

Degraded Object Oddity

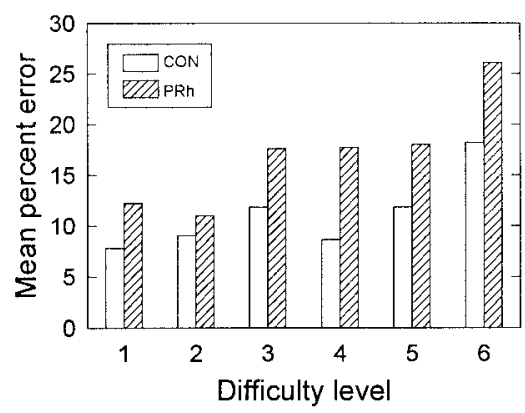

G

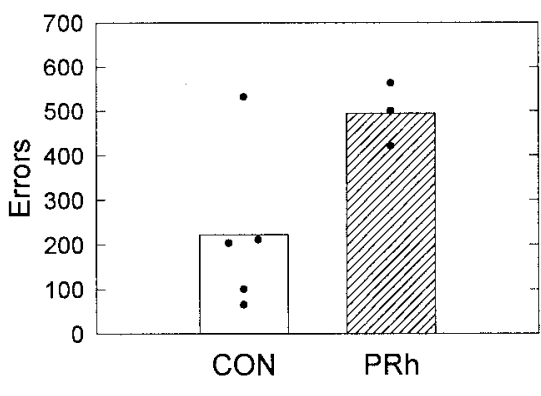

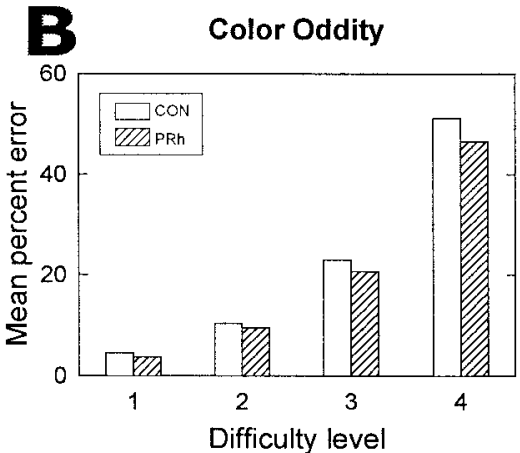
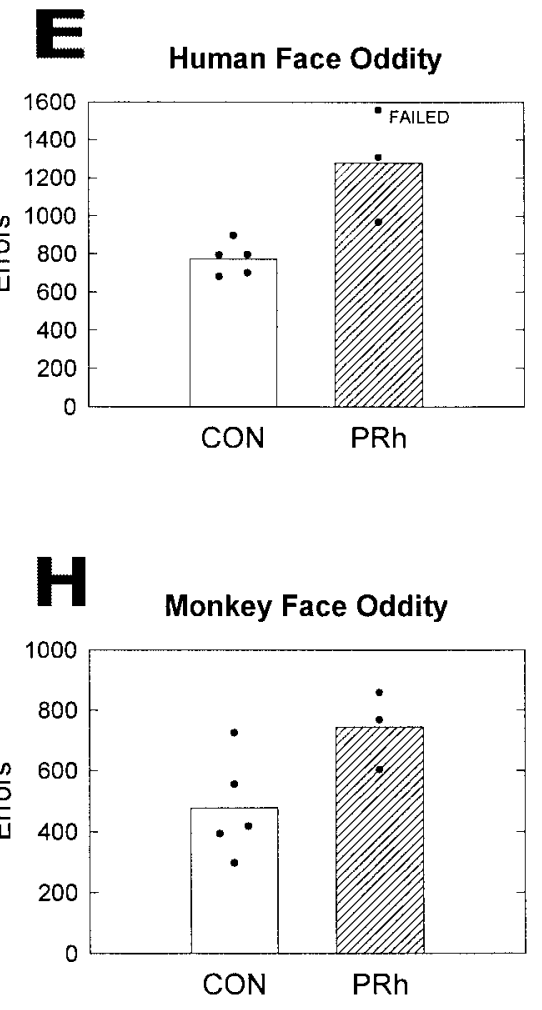

Figure 4. $A i$, Mean percentage of difference between the PRh and CON groups in terms of the difference in postoperative versus preoperative performance on problems of increasing difficulty level (1-6) in the object oddity stage of task A. Aii, Individual error scores and mean errors made by the $\mathrm{PRh}$ and $\mathrm{CON}$ group on a new set of postoperative object oddity discriminations. $B$, Mean percentage of error made by the PRh and CON groups on problems of increasing difficulty level (1-4) in task B (Color Oddity). C, Mean percentage of error made by the PRh and CON groups on problems of increasing difficulty level (1-6) in task C (Shape Oddity). D, Mean percentage of error made by the PRh and CON groups across different levels of masking (1-6) in task D (Degraded Object Oddity). E, Individual error scores and mean errors made by the PRh and CON group on the human face oddity stage of task. E, F, Mean percentage of error made by the PRh and CON groups on problems of increasing difficulty level (1-4) in task F (Size Oddity). G. Individual error scores and mean errors made by the PRh and CON groups on task G (Scene Oddity). H, Individual error scores and mean errors made by the PRh and CON groups on the monkey face oddity stage of task $\mathrm{H}$.

remained on the screen for a further $1 \mathrm{sec}$ to provide additional visual feedback for making a correct choice. Subsequently the S+ also disappeared from the screen, and an intertrial interval of $10 \mathrm{sec}$ was commenced during which the screen remained blank. Alternatively, if the subject touched one of the five incorrect identical stimuli $(\mathrm{S}-)$ then all the stimuli immediately disappeared from the screen, and an intertrial interval of $20 \mathrm{sec}$ was commenced. If the subject touched the screen during the intertrial interval, then the intertrial interval was restarted. After the touch screen had not been touched for the entire uninterrupted intertrial interval, the next trial began. Each session continued in this way using in each trial two clip art images taken from a set of 10 clip art images. The two clip art images selected in each trial were chosen at random without replacement so that no clip art image could be used again until all 10 clip art images had been used. In each trial one of the two clip art images selected was randomly assigned to be the $\mathrm{S}+$ and the other the $\mathrm{S}-$ so that during the entire session each stimulus appeared approximately equally often as an $\mathrm{S}+$ or $\mathrm{S}-$. Therefore there were no stimulus reward associations maintained throughout the trial, and the subject had no way of predicting from memory which one of the stimuli was rewarded. Instead, the subject could only reliably make a correct response by looking at the screen and making a perceptual decision that the five identical stimuli matched and could be discriminated form the one different or odd stimulus.

To facilitate learning of the oddity principle, the subjects performed one session per day during which they were required to make 100 correct responses before the lunch box opened. The lunch box contained the bulk 
of the subject's daily diet and hence provides a large motivation for the subject to continue to work through to the end of the task. Poor performance in a session results in longer intertrial intervals as well as requiring more trials to reach the criterion number of correct responses and hence delays the opening of the lunch box. The pending opening of the lunch box provides a large incentive for the subjects to concentrate on the task and maximize performance accuracy because this ensures that the lunch box opens earlier. This is in addition to the small reward pellets that are delivered during the task itself to reinforce individual correct choices.

The subjects were trained on the task using the same set of 10 stimuli each day until they attained criterion, which was set at $\geq 90 \%$ performance accuracy on that set. On the next day they were transferred to a new set of 10 different clip art images and continued daily training on this set until they reached the same criterion on this set. On the next day they were transferred to yet another set of 10 stimuli, and this process continued until the subjects had reached criterion on five different sets of 10 stimuli. Attaining criterion on the first set took on average of 25 sessions. Attaining criterion on subsequent sets took successively fewer days, demonstrating that the subjects were learning to transfer the oddity principle to new stimuli. Attaining criterion on the fifth set took only 1.5 sessions on average. At this point the subjects were deemed to have learned to perform the oddity principle, and on the next day they began training on the first experimental task.

In each of the following oddity tasks there were no associations that the subject could learn to guide their performance from trial to trial. In each task the stimuli were randomly assigned to stimulus positions so that the position of the $\mathrm{S}+$ varied from trial to trial. Each of the different stimuli in the tasks were equally likely to be used in a trial (paired and re-paired at random within the constraints of the task) and when chosen to be used were equally likely to be assigned to be the $\mathrm{S}+$ or $\mathrm{S}-$ in any particular trial. Thus accurate performance on these tasks could only be based on perceptual decisions

Task A: image oddity and object oddity. Task A commenced after completion of the oddity pretraining described above. The first stage image oddity was similar to the oddity pretraining task with clip art images described above except that the stimuli were digitized images of real objects (described in the stimulus section above). Two example trials are illustrated in Figure $3, A 1$ and $A 2$. The subjects had to select the odd image in each trial by making a perceptual judgment that five of the stimuli were identical matching views of one object and one stimulus was a view of a different object. The subjects were trained on one session per day on this task following the same procedure as described above in oddity pretraining. They continued training on this task until they had reached criterion of $\geq 90 \%$ correct responses in a single session.

On the next day the subjects began training on the second stage of task A, object oddity, which was similar to the first stage image oddity in all respects other than the fact that the five views of the $\mathrm{S}$ - were all different views rather than identical views. Two example trials are illustrated in Figure 3, $A 3$ and $A 4$. The subjects had to select the odd object in each trial by making a perceptual judgment that five of the stimuli matched in that they were different views of the same object, whereas one stimulus was a view of a different object. The subjects were trained on one session per day on this task following the same procedure as described above. They continued training on this task until they had reached criterion of $\geq 90 \%$ correct responses in a single session and then were trained for a further $3 \mathrm{~d}$.

The subjects were then assigned to groups of equivalent ability on the basis of their preoperative learning scores. Three of the subjects who were assigned to the $\mathrm{PRh}$ group received bilateral perirhinal cortex ablation at this point and were then given on average $14 \mathrm{~d}$ recovery before postoperative testing commenced. The five subjects who remained unoperated controls rested for the same period before retesting commenced.

On the next day the subjects began retesting to assess postoperative re-acquisition of the image oddity task with the same set of stimuli experienced preoperatively. They performed one session per day until reaching criterion of $\geq 90 \%$ correct responses in a single session.

On the next day the subjects began retesting to assess preoperative reacquisition of the object oddity task with the same set of stimuli experienced preoperatively. They performed one session per day until reaching criterion of $\geq 90 \%$ correct responses in a single session and then were trained for three further sessions.

On the next day the subjects began testing to assess postoperative performance on the image oddity task with a second set of 10 stimulus objects that had not been experienced before. They performed one session per day until reaching criterion of $\geq 90 \%$ correct responses in a single session.

On the next day the subjects began testing to assess postoperative performance on the object oddity task with this second set of stimuli. They performed one session per day until reaching criterion of $\geq 90 \%$ correct responses in a single session and then were trained for three further sessions.

Task B: color oddity. New postoperative training on task B (color oddity) commenced on the next day. The color oddity task was similar to the previous oddity tasks except that the stimuli were squares of different isoluminant colors (described in detail in the stimulus section above). The subjects had to select the odd-colored square in each trial by making a perceptual judgment that five of the stimuli matching in color and one stimulus was a different color. This task had a difficulty parameter that determined how similar (with respect to hue) the two colors used in any particular trial were. One color (the base color) appeared in every trial (either as the $\mathrm{S}+$ or the $\mathrm{S}-$ ), and the other color was either more red or more green than the base color. The difficulty parameter determined how much more red or how much more green the other color was with respect to the base color. The difficulty parameter was confirmed subjectively. The hardest problems (those that contained two colors closest in hue) were judged by human observers to be very hard to reliably distinguish while the easiest trials (those that contained two colors farthest apart in hue) were judged by human observers to be very easy to distinguish. For illustrative purposes only (because printed colors will appear different from the isoluminant colored stimuli on the experimental touch screens) Figure 3, B1 and $B 2$, gives an indication of what sample trials with the easiest and hardest levels of difficulty might look like, respectively. The difficulty parameter was also confirmed by the performance of the control subjects who made increasingly more errors on the increasingly difficult problems (Fig. 4B). Trials with different difficulty levels were pseudorandomly intermixed throughout the whole session. The monkeys were trained on one session per day on this task following the same procedure as described for the previous oddity tasks. They continued training on this task with one session per day for $10 \mathrm{~d}$. Thus, the subjects made in total 1000 correct responses on this task in addition to a variable number of errors.

Task C: shape oddity. New postoperative training on task C (shape oddity) commenced on the next day. The shape oddity task was similar to the previous oddity tasks except that the stimuli were polygons of varying numbers of sides (described in detail in the stimulus section above). The subjects had to select the odd polygon in each trial by making a perceptual judgment that five of the stimuli were polygons of matching shape, and one stimulus was a polygon of a different shape. This task had a difficulty parameter that determined how many sides each of the two polygons had. In every trial one of the polygons had two more sides than the other polygon, but the difficulty parameter determined the mean number of sides of these two polygons. The difficulty parameter was confirmed subjectively. Human subjects judged it harder to distinguish between polygons in problems containing polygons with more sides than in problems containing polygons with fewer sides. Figure 3, $C 1$ and $C 2$, illustrates sample trials with an easier problem (three-sided versus fivesided polygons) and a harder problem (six-sided versus eight-sided polygons), respectively. The difficulty parameter was also confirmed by the performance of the control subjects, who made increasingly more errors on the increasingly difficult problems (Fig. 4C). Trials with different difficulty levels were pseudorandomly intermixed throughout the whole session. The subjects were trained on one session per day on this task following the same procedure as described for the previous oddity tasks. They continued training on this task with one session per day for $12 \mathrm{~d}$. On the first four of these days the subjects were required to attain 50 correct responses to open the lunch box, and on the subsequent $8 \mathrm{~d}$ the subjects were required to obtain 100 correct responses to open the lunch box. Thus, the subjects made in total 1000 correct responses on this task in addition to a variable number of errors.

Task D: degraded object oddity. New postoperative training on task D (degraded object oddity) commenced on the next day. The degraded object oddity task was similar to the previous oddity tasks except that the stimuli were digitized images of objects behind a mask of random grayscale pixels. The digitized images were the same set of stimuli used in the new postoperative testing of object oddity. This task was identical to that task except for the addition of the mask. Different levels of mask obscured the objects to differing degrees (the masks are described in detail in the stimulus section above). Despite the obscuring mask the 
subjects had to select the odd object in each trial by making a perceptual judgment that five of the stimuli were different views of a matching object and one stimulus was a view of a different object. Trials with different mask levels were pseudorandomly intermixed throughout the whole session. This level of the mask was designed to be a difficulty parameter to modulate how hard it was to perceive the stimuli. The difficulty parameter was confirmed subjectively in human subjects who judged it harder to perceive the stimuli through the masks that obscured the objects to a greater degree. Figure $3, D 1$ and $D 2$, illustrates sample trials with less and greater degrees of masking, respectively. However, although there was some indication that the control subjects made more errors on trials with the most severe mask compared with trials with the least severe mask (Fig. 4D), there was not a statistically significant linear trend of increasing error rate made by controls with increasing mask level. Thus, increasing the level of the mask parameter in this task may not increase in a linear manner the difficulty that the subjects in this experiment have in perceiving the objects through the increasing levels of the mask. The monkeys were trained on one session per day on this task following the same procedure as described for the previous oddity tasks. They continued training on this task with one session per day for $7 \mathrm{~d}$. Thus, the subjects made in total 700 correct responses on this task in addition to a variable number of errors.

Task E: human face oddity. New postoperative training on task E (human face oddity) commenced on the next day. The human face oddity task was similar to the previous oddity tasks except that the stimuli were digitized images of human faces (described in detail in the stimulus section above). In the first stage "human face image oddity", the subjects had to select the odd stimulus in each trial by making a perceptual judgment that five of the faces were identical views of a matching face and the other stimulus was a view of a different face. Two example trials are illustrated in Figure 3,E1 and E2. The subjects were trained on this task following the same procedure as described for the previous oddity tasks. They performed one session per day until reaching criterion of $\geq 90 \%$ correct responses in a single session.

On the next day the subjects began training on the second stage of task E, "human face oddity", which was similar to the first stage human face image oddity in all respects other than the fact that the five views of the $\mathrm{S}-$ were all different views rather than identical views of that face. Two example trials are illustrated in Figure 3,E3 and E4. The subjects had to select the odd individual in each trial by making a perceptual judgment that five of the stimuli matched in that they were different views of the same individual and one stimulus was a view of a different individual. The subjects were trained on this task following the same procedure as described for the previous oddity tasks. They performed one session per day until reaching criterion of $\geq 90 \%$ correct responses in a single session.

Task F: size oddity. New postoperative training on task F size oddity commenced on the following day. The size oddity task was similar to the previous oddity tasks, except that the stimuli were outlines of square of varying size (described in detail in the stimulus section above). The subjects had to select the odd stimulus in each trial by making a perceptual judgment that five of the stimuli were the same size, and the other stimulus was a different size. This task had a difficulty parameter that determined how similar in size the two squares were. The difficulty parameter was confirmed subjectively. Human subjects judged it increasingly harder to distinguish between squares as the difference in size decreased. Figure 3, F1 and $F 2$, illustrates sample trials with one of the easiest and hardest problems, respectively. The difficulty parameter was also confirmed by the performance of the control subjects, who made more errors as the problem difficulty parameter increased (Fig. $4 F$ ). Trials with different difficulty levels were pseudorandomly intermixed throughout the whole session. The subjects were trained on this task following the same procedure as described for the previous oddity tasks. They continued training on this task with one session per day for $7 \mathrm{~d}$. Thus, the subjects made in total 700 correct responses on this task in addition to a variable number of errors.

Task G: scene oddity. New postoperative training on task $G$ scene oddity commenced on the next day. The scene oddity task was similar to the previous oddity tasks except that the stimuli were digitized images of scenes. The stimuli are described in detail in the stimulus section above, and two example trials are illustrated in Figure 3, G1 and G2. The subjects had to select the odd scene in each trial by making a perceptual judgment that three of the stimuli were the same scene and the other stimulus was a different scene. The subjects were trained on this task following the same procedure as described for the previous oddity tasks.
They performed one session per day until reaching criterion of $\geq 90 \%$ correct responses in a single session or until a maximum of 15 sessions had been completed.

Task H: monkey face oddity. New postoperative training on the task $\mathrm{H}$ monkey face oddity commenced on the next day. The monkey face oddity task was similar to the previous oddity tasks except that the stimuli were digitized images of monkey faces (described in the stimulus section above). In the first stage, monkey face image oddity, the subjects had to select the odd stimulus in each trial by making a perceptual judgment that three of the four faces were identical views of a matching face, and one stimulus was a view of a different face. Two example trials are illustrated in Figure 3, $H 1$ and $H 2$. The subjects were trained on this task following the same procedure as described for the previous oddity tasks. They performed one session per day until reaching criterion of $\geq 90 \%$ correct responses in a single session or until a maximum of 15 sessions had been completed.

On the next day the subjects began training on the second stage of task $\mathrm{H}$, monkey face oddity which was similar to the first stage monkey face image oddity in all respects other than the fact that the three views of the $\mathrm{S}-$ were all different views rather than identical views of that monkey's face. Two example trials are illustrated in Figure 3, H3 and H4. The subjects had to select the odd individual in each trial by making a perceptual judgment that three of the four stimuli matched in that they were different views of the same monkey's face and one stimulus was a view of a different monkey's face. The subjects were trained on this task following the same procedure as described for the previous oddity tasks. They performed one session per day until reaching criterion of $\geq 90 \%$ correct responses in a single session.

This completed the behavioral testing in this study. After completion of subsequent behavioral tasks as part of a separate study to be reported elsewhere the three subjects in the PRh group were perfused, and histology to verify the extent of their lesions was obtained.

\section{RESULTS}

\section{Task A: image oddity and object oddity}

\section{Analysis of preoperative performance}

Preoperatively the CON group made a mean of five errors learning the image oddity task to criterion, and the PRh group made a mean of seven errors. There was no significant difference between the performance of the groups on this stage of training (independent samples test: $t=-1.368$; df $=6 ; p=0.2$; NS). Preoperatively the CON group made a mean of 65 errors learning the object oddity task to criterion, and the $\mathrm{PRh}$ group made a mean of 129 errors. There was no significant difference between the performance of the groups on this stage of training (independent samples test: $t=-1.442$; df $=6 ; p=0.2$; NS). Thus, the two groups were satisfactorily matched for preoperative performance.

\section{Analysis of postoperative reacquisition}

Postoperatively the CON group made a mean of 29 errors relearning the image oddity task to criterion, and the PRh group made a mean of only 14 errors. There was no significant difference between the performance of the groups on this stage of training (independent samples test: $t=1.119$; df $=6 ; p=0.3$; NS). This result is consistent with our hypothesis that the PRh group should not be impaired on discriminating between two stimuli, even if they are objects, when the stimuli differ in respect to many simple and moderately complex features.

Postoperatively the CON group made a mean of 121 errors relearning the object oddity task to criterion, and the PRh group made a mean of 143 errors. Considering postoperative performance alone on the performance of both groups averaged over all the different problems, there was no significant difference in performance between the groups (independent samples test: $t=$ -0.373 ; df $=6 ; p=0.7$; NS).

However, our hypothesis predicts that only the more difficult problems that require the subject to discriminate at an object 


\begin{tabular}{|c|c|c|c|c|c|c|c|c|c|}
\hline Task and measure analyzed & CON1 & CON2 & CON3 & CON4 & CON5 & PRh-1 & PRh-2 & PRh-3 & Impaired \\
\hline \multicolumn{10}{|l|}{ Object oddity reacquisition (post-op minus pre-op mean } \\
\hline$\%$ error across all levels) & -1.2 & -1.7 & -0.7 & -1.7 & -1.8 & 0.7 & 1.5 & 4.3 & Yes \\
\hline New post-op image oddity (errors to criterion) & 17 & 1 & 20 & 16 & 23 & 9 & 5 & 7 & No \\
\hline New post-op object oddity (errors to criterion) & 142 & 104 & 183 & 273 & 322 & 590 & 277 & 491 & Yes \\
\hline Color oddity (mean \% error across all levels) & 27.7 & 18.9 & 17.6 & 28.1 & 32.8 & 28.0 & 19.3 & 12.9 & No \\
\hline Shape oddity (mean $\%$ error across all levels) & 33.7 & 35 & 35.8 & 32.3 & 39.7 & 38.2 & 31.7 & 35.8 & No \\
\hline Degraded object oddity (mean $\%$ error across all levels) & 12.1 & 7.6 & 9.2 & 13.8 & 14.2 & 21.5 & 15.8 & 14.1 & Yes \\
\hline Human face image oddity (errors to criterion) & 90 & 97 & 110 & 379 & 272 & 2020F & 107 & 274 & No \\
\hline Human face oddity (errors to criterion) & 680 & 700 & 796 & 896 & 793 & $1558 \mathrm{~F}$ & 1308 & 967 & Yes \\
\hline Size oddity (mean \% error across all levels) & 34.7 & 28.6 & 33.1 & 42.2 & 34.5 & 36.6 & 36.1 & 35.3 & No \\
\hline Scene oddity (errors to criterion) & 211 & 65 & 99 & 532 & 203 & 500 & 421 & 563 & Yes \\
\hline Monkey face image oddity (errors to criterion) & 271 & 143 & 217 & 819 & 105 & 1323 & 279 & 534 & No \\
\hline Monkey face oddity (errors to criterion) & 555 & 298 & 417 & 725 & 393 & 604 & 859 & 768 & Yes \\
\hline
\end{tabular}

$F$ denotes failure to reach criterion

level should be impaired after perirhinal cortex ablation. Thus, to compare the relative effect of perirhinal cortex ablation on problems of differing difficulty, we ranked the performance of each monkey on each of the different problems preoperatively. Random pairing of the 10 objects in the set creates 90 different problems, and we recorded the preoperative error rate on each of these 90 different problems. For each monkey we ranked the difficulty of the 90 problems by the preoperative error rate and assigned each of these problems to one of six levels of problem difficulty. The error rate for each problem was recorded postoperatively so that we could compare performance preoperatively and postoperatively for each subject across six levels of problem difficulty. This was done individually for each monkey because different monkeys found some problems harder than other problems.

\section{Analysis of postoperative reacquisition relative to preoperative performance}

The mean differences in performance between the two groups across different levels of our difficulty parameter are illustrated in Figure $4 A i$ (see Table 1 for individual scores averaged across difficulty levels). Analysis of this difference in postoperative performance relative to preoperative performance showed that the PRh group was highly significantly impaired relative to that of the CON group [repeated measures ANOVA: group, $F=11.1$; $\mathrm{df}=$ $(1,6) ; p=0.016]$. Figure $4 A i$ also shows that the mean difference in postoperative performance relative to preoperative performance between the PRh group, and the CON group tends to increase as problem difficulty increases. Analysis of the linear trend of increase in this deficit with respect to our difficulty parameter did not quite attain statistical significance in a onetailed test [repeated measures ANOVA: group $\times$ difficulty-level, linear trend, $F=2.776$; df $=(1,6) ; p=0.07$; one-tailed]. This may be attributable to the fact that our difficulty parameter simply ranked problems, and therefore different levels of this parameter did not necessarily represent regular increases in difficulty. However, Figure $4 A i$ clearly shows that the deficit in performance of the $\mathrm{PRh}$ group relative to the $\mathrm{CON}$ group reaches its greatest level and is quite considerable on the hardest problems.

\section{Analysis of new postoperative performance}

Postoperatively the CON group made a mean of 15 errors learning the image oddity task with a new set of stimuli to criterion, and the PRh group made a mean of seven errors (see Table 1 for individual scores). There was no significant difference between the performance of the groups on this stage of training (independent samples test: $t=-1.634$; df $=6 ; p=0.15$; NS). This result is consistent with our hypothesis that the PRh group should not be impaired on discriminating between two stimuli, even if they are objects, when the stimuli differ in respect to many simple and moderately complex features.

Postoperatively the CON group made a mean of 205 errors learning the object-oddity task with a new set of stimuli to criterion, and the PRh group made a mean of 453 errors (see Table 1 for individual scores). These data are illustrated in Figure 4 Aii. Because this new set of stimuli was not experienced preoperatively, we had no preoperative measure of what particular problems the PRh group would have found difficult so no postoperative versus preoperative comparison can be made. Instead we analyzed the mean errors averaged over all problems. The PRh group was significantly impaired relative to the CON group on this stage of training (independent samples test: $t=2.866$; $\mathrm{df}=6 ; p=0.029)$. Thus, the new postoperative object oddity task was significantly impaired after perirhinal cortex ablation. Because the CON group made significantly more errors on the new postoperative object oddity problem set then on postoperative reacquisition of the preoperatively learned object oddity problem set (paired samples test: $t=-6.025$; df $=4 ; p=0.004$ ), we can conclude that solving the new postoperative problems was harder. Thus the PRh deficit on this new set as a whole can be understood as reflecting the increased difficulty that this set may have presented in terms of requiring the subjects to base their discriminations between the objects on discriminations at an object level rather than discriminations at a simple feature or moderately complex feature level.

\section{Task B: color oddity}

For each subject we calculated the mean percentage of error made to each of the four different levels of problem difficulty (problem difficulty was determined by the relative difference in color of the base color from the second color present along either the red or green axis). The data for postoperative performance on the color oddity task are illustrated in Figure $4 B$ (see Table 1 for individual scores averaged across difficulty levels). Analysis of the data shows that there was no interaction between the between-subjects factor, "group" and the within-subjects factor 
"difficulty level" [repeated measures ANOVA: group $\times$ difficulty level, $F=1.95$; df $=(3,18) ; p=0.18$; NS], nor was there a significant main effect of group [repeated measures ANOVA: group, $F=3.25$; $\mathrm{df}=(1,6) ; p=0.12$; NS]. Thus, the PRh group is not impaired at perceptually discriminating between squares of different colors even when the task is very difficult.

\section{Task C: shape oddity}

For each subject we calculated the mean percentage of error made to each of the six different levels of problem difficulty. The data for postoperative performance on the shape oddity task are illustrated in Figure $4 C$ (see Table 1 for individual scores averaged across difficulty levels). Analysis of the data shows that there was no interaction between the between-subjects factor, "group" and the within-subjects factor, "difficulty level" [repeated measures ANOVA: group $\times$ difficulty level, $F<1$; df $=(5,30)]$, nor was there a significant main effect of group [repeated measures ANOVA: group, $F<1$; df $=(1,6)]$. Thus, the PRh group is not impaired at perceptually discriminating between polygons of different shape even when the task is very difficult.

\section{Task D: degraded object oddity}

For each subject we calculated the mean percentage of error made to problems under the six different levels of obscuring mask. The data for postoperative performance on the degraded object oddity task are illustrated in Figure $4 D$ (see Table 1 for individual scores averaged across levels of mask). Analysis of the data shows that the interaction between the between-subjects factor, "group" and the within-subjects factor, "difficulty level" linear trend was not quite significant in a one-tailed test [repeated measures ANOVA: group $\times$ difficulty level linear trend, $F=3.237$; df $=$ $(1,6) ; p=0.06$; one-tailed]. However, the PRh group was significantly impaired relative to the CON group across all levels of mask [repeated measures ANOVA: group, $F=5.584$; df $=(1,6)$; $p=0.028$; one-tailed]. There was also a significant main effect of mask level [repeated measures ANOVA: mask level, $F=18.074$; df $=(5,30) ; p<0.001]$.

This task used the same set of object stimuli as the new postoperative object oddity stage of task 1 in which the $\mathrm{PRh}$ group was shown to be impaired previously. This analysis shows that the PRh group remain impaired on tasks that involve making perceptual discriminations between objects on the basis of discriminating at the level of objects.

Caution needs to be exercised before concluding that increasing the mask does not increase the size of the deficit of the PRh group because the interaction term is almost significant. However, averaged across all levels and all problems the magnitude of the impairment of the PRh group on this degraded object oddity task is very similar to the magnitude of the impairment of the PRh group on the object oddity task without the mask. Thus, imposing a mask on the object oddity task does seem to make the task harder for all subjects in a perirhinal-independent manner.

\section{Task E: human face oddity}

The CON group made a mean of 190 errors learning the human face image oddity stage of task 5 to criterion, and the PRh group made a mean of 800 errors (see Table 1 for individual scores). One subject in the PRh group (PRh1) was deemed to have failed to learn the human face image oddity task after making $>2000$ errors in 30 sessions without attaining criterion. Therefore, we used a nonparametric test to analyze the mean error data. There was no significant difference between the performance of the groups on this stage of training (Mann-Whitney independent samples rank sum test: $U=4 ; p=0.3$; NS).

The CON group made a mean of 773 errors learning the human face oddity stage of task 5 to criterion, and the PRh group made a mean of 1278 errors (see Table 1 for individual scores). The data for postoperative performance on the human face oddity task are illustrated in Figure $4 E$. One subject in the $\mathrm{PRh}$ group (PRh1) was deemed to have failed to learn the human face oddity task after making $>1500$ errors in 32 sessions without attaining criterion. Therefore, we used a nonparametric test to analyze the mean error data. There was a highly significant difference between the performance of the groups on this stage of training because all of the PRh group performed worse than any of the CON group (Mann-Whitney independent samples rank sum test: $U<0.001 ; p=0.036)$.

Thus, the PRh group was significantly impaired relative to the $\mathrm{CON}$ group on human face oddity stage of task $\mathrm{E}$ but was unimpaired on the human face image oddity stage of task E. This parallels the pattern of impairment on the earlier object image oddity and object oddity tasks.

\section{Task F: size oddity}

For each subject we calculated the mean percentage of error made to each of the four different levels of problem difficulty. The data for postoperative performance on the size oddity task are illustrated in Figure $4 F$ (see Table 1 for individual scores averaged across difficulty levels). Analysis of the data shows that there was no interaction between the between-subjects factor "group" and the within-subjects factor "difficulty level" [repeated measures ANOVA: group $\times$ difficulty level, $F=1.478$; df $=(3,18)$; $p=0.25$; NS]. Nor was there a significant main effect of group [repeated measures ANOVA: group, $F<1$; df $=(1,6)]$. Thus, the $\mathrm{PRh}$ group is not impaired at perceptually discriminating between squares of different sizes even when the task is very difficult.

\section{Task G: scene oddity}

The CON group made a mean of 222 errors learning the scene oddity stage of task 7 to criterion, and the $\mathrm{PRh}$ group made a mean of 495 errors (see Table 1 for individual scores). The data for postoperative performance on the scene oddity task are illustrated in Figure $4 G$. The PRh group was significantly impaired relative to the CON group (independent samples test: $t=-2.39$; $\mathrm{df}=6 ; p=0.027$; one-tailed).

One may speculate regarding whether the monkeys view each one of the four scenes in every trial this task as a whole scene containing objects on a background or whether the monkeys view each digitized photograph of a scene as an object in itself. In either case it seems likely that the ability to process stimuli at an object level would facilitate performance on this task and is consistent with the impairment on this task.

\section{Task H: monkey face oddity}

The CON group made a mean of 311 errors learning the monkey face image oddity stage of task 8 to criterion, and the PRh group made a mean of 712 errors (see Table 1 for individual scores). However, the $\mathrm{PRh}$ group was not significantly impaired relative to the CON group on the monkey face image oddity stage of task 8 (independent samples test: $t=-1.393$; df $=6 ; p=0.2$; NS).

The CON group made a mean of 478 errors learning the monkey face oddity stage of task 5 to criterion, and the PRh group made a mean of 744 errors (see Table 1 for individual scores). The data for postoperative performance on the human 
face oddity task are illustrated in Figure $4 H$. The PRh group was found to be significantly impaired relative to the CON group on the monkey face oddity stage of task 8 (independent samples test: $t=-2.354 ; \mathrm{df}=6 ; p=0.029$; one-tailed).

Thus, the $\mathrm{PRh}$ group was significantly impaired relative to the $\mathrm{CON}$ group on monkey face oddity stage of task $\mathrm{H}$ but was unimpaired on the monkey face image oddity stage of task $\mathrm{H}$. This parallels the pattern of impairment on the earlier object image oddity and object oddity tasks and also the earlier human face image oddity and human face oddity tasks.

\section{Summary of results}

In task 1 the PRh group was impaired relative to the CON group at hard object oddity problems but unimpaired at easier object oddity problems and image oddity problems. Thus, the PRh group was impaired relative to the $\mathrm{CON}$ group at making perceptual discriminations when the stimuli were objects and the discriminanda were all of different views and the discrimination could not easily be solved on the basis of simple or moderately complex features of objects. Because the PRh group was subsequently impaired on several further tasks, we can rule out that this impairment is only a relatively transient effect.

The PRh group remained completely unimpaired on very difficult color oddity problems (task B), very difficult shape oddity problems (task C), and very difficult size oddity problems (task F). Thus, we can rule out that the impairment may have been simply related to the perceptual difficulty of the discrimination.

The PRh group was impaired on degraded object oddity problems (task D), human face oddity problems (task D), scene oddity problems (task $\mathrm{G}$ ), and monkey face oddity problems (task $\mathrm{H}$ ). The PRh group remained unimpaired on human face image oddity problems (control stage for task D) and monkey face image oddity problems (control stage for task $\mathrm{H}$ ). Therefore, taken together the pattern of impaired and unimpaired performance on every task shows that the nature of the perceptual impairment after PRh cortex damage is selective. Only problems that require perceptual discrimination at a more abstract level (such as at the level of an object) are impaired, whereas problems that can be solved purely by simple or moderately complex feature discrimination are unimpaired (regardless of whether the problems involve objects).

\section{DISCUSSION}

We trained monkeys to make perceptual discriminations to choose the one stimulus of several stimuli that were presented at the same time on the touch screen that was the odd stimulus. After learning the principle, the monkeys were able to easily transfer the principle to a range of different types of stimuli. We used this paradigm to assess whether after bilateral perirhinal cortex ablation there was any impairment in making perceptual discriminations, and if so, whether the impairment was selective to particular types of stimuli. The results from every stage of every task in this study are fully consistent with our hypothesis that the macaque perirhinal cortex contributes to making perceptual discriminations between stimuli when the discrimination requires processing of the stimuli to a more abstract level, such as at the level of an object, but not to perceptual discriminations that can be done on the basis of discriminating between simple or only moderately complex features of objects. Therefore, we conclude that role of the perirhinal cortex in the monkey extends beyond stimulus memory and contributes to object perception.

This is consistent with evidence from earlier lesion studies.
Eacott et al. (1994) found that simultaneous matching-to-sample was impaired after rhinal cortex ablations. Buckley and Gaffan (1997, 1998b,c) found that perirhinal lesions only impaired concurrent object discrimination learning tasks that placed high demands on object identification. Tasks with few problems that could be solved on the basis of simple feature discrimination alone were unimpaired, whereas tasks with many problems that likely required discrimination between object representations were impaired (Buckley and Gaffan, 1997). Likewise, tasks with small stimulus sets but with many distracting foils in each problem (Buckley and Gaffan, 1997), with stimuli presented in different familiar views between trials (Buckley and Gaffan, 1998b), with stimuli presented in new views (Buckley and Gaffan, 1998c), and with familiar stimuli presented in new scenes (Buckley and Gaffan, 1998c) were all impaired. Each of these tasks requires the representation of the discriminandum to be more specific. The same pattern of results is found in object recognition memory tasks as Eacott et al. (1994) showed that delayed matching-tosample was impaired with large, but not small, stimulus sets. Saksida and Bussey (1998) implemented a neural network model of IT function and lesioned the "feature conjunction layer" of their model corresponding to the perirhinal cortex. Perirhinal lesions in this model replicated several of the effects of perirhinal lesions in monkeys, including the set size effect in concurrent discrimination learning (Buckley and Gaffan, 1997) and the deficit in configural learning despite small set sizes (Buckley and Gaffan, 1998a). Their model (Saksida and Bussey, 1999) also predicted the greater degree of deficit after perirhinal cortex ablation found in discriminating morphed stimuli when the degree of feature overlap was increased (Bussey and Saksida, 1999). Taken together, this pattern of impairments in lesion studies across a range of memory tasks suggests that the role of the perirhinal cortex in the monkey extends beyond stimulus memory (for review, see Murray and Bussey, 1999; Buckley and Gaffan, 2000; Murray and Richmond, 2001).

This study confirms that the perirhinal cortex does indeed contribute to perception but that it plays a specific role in perception. It contributes when stimuli have to be processed to a more abstract level such as at the level of objects. Perirhinal lesions also disrupt configural learning (Buckley and Gaffan, 1998a), a task that can only be solved by associating specific combinations of features, not individual features themselves, with reward. Objects consist of configural arrangements of spatially contiguous features and therefore object recognition requires analysis of the configural arrangement of these features. Thus, the role of the perirhinal cortex in discriminating visual configural cues is closely related to the general role of the perirhinal cortex in discriminating multiple individual objects.

Anatomical evidence also supports the notion that the perirhinal cortex is specialized for processing objects. Saleem and Tanaka (1996) indicated that sites in the perirhinal cortex receive convergent inputs from multiple widely distributed sites in the ventral part of anterior TE. Thus, different moderately complex features of objects represented by distant columns in TE (for review, see Tanaka, 1996) may be associated together in the perirhinal cortex to represent whole objects. In addition to the prominent inputs from unimodal visual areas TEO and TE, the perirhinal cortex receives projections from diverse unimodal and polymodal areas of association cortex (Suzuki and Amaral, 1994a) and is associated with subcortical structures such as the medial basal nucleus of the amygdala (Cheng et al., 1997). Thus, the perirhinal cortex is in a position to associate together infor- 
mation about individual objects from diverse sources, consistent with the proposed specialized role for the perirhinal cortex in processing polymodal representations of multiple individual objects (for review, see Buckley and Gaffan, 2000).

Electrophysiological evidence also supports the notion that the perirhinal cortex is specialized for processing objects. Brown et al. (1987) demonstrated that some neurons in the anterior inferior temporal cortex and perirhinal cortex showed recognitionrelated responses because they respond more strongly to the first than to subsequent presentations of unfamiliar objects. It was proposed that these decremental responses, alternatively described as "adaptive filtering" (Li et al., 1993; Miller et al., 1993) or "stimulus-specific adaptation" (Sobotka and Ringo, 1993, 1994; Ringo, 1996) might constitute the neural basis of object recognition memory, suggesting a role in mnemonic processing. However, a majority of recorded cells in the perirhinal cortex show stimulus selectivity without showing decremental responses. Furthermore, recognition memory performance has been dissociated from decremental responses (Miller and Desimone, 1993; Sobotka and Ringo, 1996; Tang et al., 1997). Thus, a range of neuronal mechanisms exist in the perirhinal cortex perhaps contributing to a range of behaviors. Recognition memory itself for instance requires both stimulus identification as well as judgments about previous occurrence. Thus, electrophysiological evidence is also consistent with the perirhinal cortex being specialized for processing information about objects and not specialized for purely mnemonic or purely perceptual processes.

Higuchi and Miyashita (1996) showed that rhinal cortex lesions disrupted the association of paired visual stimuli (fractal patterns) previously recorded in TE. Thus, the perirhinal cortex may associate together stimuli including features of objects and direct the storage of these associations into respective areas of association cortex. Booth and Rolls (1998) reported that a small proportion of cells in TE responded in a view invariant manner to objects, and it was proposed (Rolls, 2000) that the perirhinal cortex might also help to build such invariant perceptual representations of objects by providing the necessary short-term memory component (Holscher and Rolls, 2001) or the necessary anatomical convergence for the TE neurons with responses to different views of features of objects to be linked together by association to form an (invariant) object representation (or "concept"). Consistent with the idea that the perirhinal cortex directs the association together of information stored in TE, Naya et al. (2001) reported that a memory-retrieval signal appeared in perirhinal cortex before TE, and then TE neurons were gradually recruited to represent the sought target. Further behavioral evidence that the perirhinal cortex at least maintains associations between information stored elsewhere is that damage to the perirhinal region typically produces more severe retrograde memory deficits than deficits in new associative learning (Gaffan and Murray, 1992; Buckley and Gaffan, 1997; Thornton et al., 1997). The current study implies that the perirhinal cortex is required to maintain representations of familiar objects, as well as build representations of new objects, in perceptual tasks too.

In short there is overwhelming evidence to support the idea that the perirhinal cortex is specialized for processing objects and that the specialization extends beyond stimulus memory to include stimulus perception. Nevertheless, some authors have recently maintained that the perirhinal cortex of the monkey is not involved in perception (Buffalo et al., 1999, 2000). These authors have dismissed the contrary evidence from many of the monkey lesion studies discussed above by claiming that these deficits were attributable to TE damage. This argument can be refuted because none of the perirhinal lesions reported by Buckley and colleagues encroached both substantially and bilaterally into adjacent TE. These authors also claim that monkeys show intact performance on immediate, but not delayed, recognition memory tasks, thus arguing that perception but not memory is intact. However, although this may provide evidence for a role for the perirhinal cortex in memory, a lack of deficit on immediate matching does not rule out a role for the perirhinal cortex in perception, unless the discriminandum are required to be discriminated at an object level or there is high feature overlap between the stimuli. Buffalo et al. (2000) also report that unlike perirhinal lesioned monkeys, TE lesioned monkeys are unable to acquire a nonmatching rule postoperatively, thus arguing that that TE has a greater role in perceptual discrimination. However, this may simply reflect the greater disruption that the larger TE lesion has on acquiring the nonmatching rule itself rather than a greater role for TE in discriminating between stimuli. Furthermore, because cells in TE appear to be specialized for processing moderately complex object features and not objects (Tanaka, 1996), it would be expected that TE, not perirhinal cortex, would be recruited for perceptual discriminations that can be done on the basis of moderately complex object features. Therefore, Buffalo et al. (2000) did not address the role of the perirhinal cortex in making perceptual discriminations between stimuli at an object level.

Finally, Stark and Squire (2000) recently used some of these oddity tasks to assess whether encephalitic patients with extensive medial temporal lobe damage including the perirhinal cortex were impaired at making perceptual discriminations. Because these patients showed no impairments, they concluded that the perirhinal cortex of humans does not contribute to perception and that the most likely explanation for the discrepancy in results between humans and monkeys may be a different role of the perirhinal cortex between species. However, there are other interpretations. Our hypothesis predicts that subjects with perirhinal cortex damage should only be impaired at making perceptual discriminations between stimuli when these discriminations require processing of the stimuli at the level of an object, and the human subjects in this study may not have done this. Unlike monkeys, the human patients had no experience with the real objects to facilitate recognition of these kinds of object from different digitized views. The patients were elderly and may have been unfamiliar with the concept of recognizing objects from different views presented on a computer screen. Indeed, human controls found the degraded object oddity task far harder than any of the control monkeys. Our degraded object oddity analysis shows that simply making the objects harder to see does not increase the demands on the perirhinal cortex. Rather, to show an effect in human patients with damage to this region one should perhaps ensure that the stimuli are processed as objects while at the same time being harder to discriminate from one another which is currently under investigation. Finally, the possibility remains that the cortical region specialized for processing stimuli at an object level in humans may be located more posteriorly than in monkeys.

To conclude the perirhinal cortex of the monkey contributes to object perception and memory. It is inappropriate to characterize the perirhinal cortex as either a uniquely visual or memory structure. Likewise, other areas in the ventral processing stream and limbic system may also contribute to perception and memory of the stimuli they process. 


\section{REFERENCES}

Amaral DG, Insausti R, Cowan WM (1987) The entorhinal cortex of the monkey. I. Cytoarchitectonic organization. J Comp Neurol 230:465-496.

Booth MCA, Rolls ET (1998) View-invariant representations of familiar objects by neurons in the inferior temporal visual cortex. Cereb Cortex $8: 510-523$.

Brodmann K (1909) Vergleichende lokalisationslehre der grosshirnrinde in ihren prinzi pen dargestellt auf grund des zellenbaus. Liepzig: Barth.

Brown MW, Wilson FA, Riches IP (1987) Neuronal evidence that inferomedial temporal cortex is more important than hippocampus in certain processes underlying recognition memory. Brain Res 409:158-162.

Buckley MJ, Gaffan D (1997) Impairment of visual objectdiscrimination learning after perirhinal cortex ablation. Behav Neurosci 111:467-475.

Buckley MJ, Gaffan D (1998a) Perirhinal cortex ablation impairs configural learning and paired-associate learning equally. Neuropsychologia 36:535-546.

Buckley MJ, Gaffan D (1998b) Learning and transfer of object-reward associations and the role of the perirhinal cortex. Behav Neurosci 112:15-23.

Buckley MJ, Gaffan D (1998c) Perirhinal cortex ablation impairs visual object identification. J Neurosci 18:2268-2275.

Buckley MJ, Gaffan D (2000) The hippocampus, perirhinal cortex and memory in the monkey. In: Brain, perception, memory: advances in cognitive neuroscience (Bolhuis JJ, ed), pp 279-298. Oxford: Oxford UP.

Buckley MJ, Gaffan D, Murray EA (1997) Functional double dissociation between two inferior temporal cortical areas: perirhinal cortex versus middle temporal gyrus. J Neurophysiol 77:587-598.

Buckley MJ, Booth MCA, Rolls ET, Gaffan D (1998) Selective visualperceptual deficits following perirhinal cortex ablation in the macaque. Soc Neurosci Abstr 24:18.

Buffalo B, Ramus SJ, Squire LR, Zola (2000) Perception and recognition memory in monkeys following lesions of area TE and perirhinal cortex. Learn Mem 7:375-382.

Buffalo EA, Reber PJ, Squire LR (1998) The human perirhinal cortex and recognition memory. Hippocampus 8:330-339.

Buffalo EA, Ramus SJ, Clark RE, Teng E, Squire LR, Zola SM (1999) Dissociation between the effects of damage to perirhinal cortex and area TE. Learn Mem 6:572-599.

Bussey TJ, Saksida LM (1999) Overgeneralization in monkeys with perirhinal cortex lesions. Soc Neurosci Abstr 25:789.

Cheng K, Saleem KS, Tanaka K (1997) Organization of corticostriatal and corticoamygdalar projections arising from the anterior inferotemporal area TE of the macaque monkey: a Phaseolus vulgaris leucoagglutinin study. J Neurosci 17:7902-7925.

DeValois RL, Morgan HC, Polson MC, Mead WR, Hull EM (1974) Psychophysical studies of monkey vision. I. Macaque luminosity and color vision tests. Vision Res 14:53-67.

Eacott MJ, Gaffan D, Murray EA (1994) Preserved recognition memory for small sets, and impaired stimulus identification for large sets, following rhinal cortex ablations in monkeys. Eur J Neurosci 6:1466-1478.

Gaffan D (1994) Dissociated effects of perirhinal cortex ablation, fornix transection and amygdalectomy: evidence for multiple memory systems in the primate temporal lobe. Exp Brain Res 99:411-422.

Gaffan D, Murray EA (1992) Monkeys (Macaca fascicularis) with rhinal cortex ablations succeed in object discrimination learning despite 24-hr intertrial intervals and fail at matching to sample despite double sample presentations. Behav Neurosci 106:30-38.

Gaffan D, Saunders RC, Gaffan EA, Harrison S, Shields C, Owen MJ (1984) Effects of fornix transection upon associative memory in monkeys: role of the hippocampus in learned action. Q J Exp Psychol B 36:173-221.

Higuchi S, Miyashita Y (1996) Formation of mnemonic neuronal responses to visual paired associates in inferotemporal cortex is impaired by perirhinal and entorhinal lesions. Proc Natl Acad Sci USA 93:739-743.

Holscher C, Rolls ET (2001) Perirhinal cortex neurons in macaques with activity related to the long-term familiarity of visual stimuli, and to working memory. Soc Neurosci Abstr 27:455.9.

Insausti R, Amaral DG, Cowan WM (1987) The entorhinal cortex of the monkey. II. Cortical afferents. J Comp Neurol 264:356-395.

Lee BB (1991) Spectral sensitivity in primate vision. In: Vision and visual dysf unction: limits of vision (Kulikowski JJ, Walsh V, Murray IJ, eds), pp 191-199. London: Macmillan.

Li L, Miller EK, Desimone R (1993) The representation of stimulus familiarity in anterior inferior temporal cortex. $\mathrm{J}$ Neurophysiol 69:1918-1929.

Miller EK, Desimone R (1993) Scopolamine affects short-term memory but not inferior temporal neurons. NeuroReport 4:81-84.

Miller EK, Li L, Desimone R (1993) Activity of neurons in anterior inferior temporal cortex during a short-term memory task. J Neurosci 13:1460-1478.

Murray EA, Bussey TJ (1999) Perceptual-mnemonic function of the perirhinal cortex. Trends Cogn Sci 3:142-151.

Murray EA, Richmond BJ (2001) Role of perirhinal cortex in object perception, memory, and associations. Curr Opin Neurobiol 11:188-193.

Naya Y, Masatoshi Y, Miyashita Y (2001) Backward spreading of memory-retrieval signal in the primate temporal cortex. Science 291:661-664.

Ringo JL (1996) Stimulus specific adaptation in inferior temporal and medial temporal cortex of the monkey. Behav Brain Res 76:191-197.

Rolls ET (2000) Functions of the primate temporal lobe cortical visual areas in invariant visual object and face recognition. Neuron 27:205-218.

Saksida LM, Bussey TJ (1998) Toward a neural network model of visual object identification in primate inferotemporal cortex. Soc Neurosci Abstr 24:1906.

Saksida LM, Bussey TJ (1999) Perirhinal cortex and stimulus overgeneralization: predictions of a neural network model. Soc Neurosci Abstr 25:789.

Saleem KS, Tanaka K (1996) Divergent projections from the anterior inferotemporal area TE to the perirhinal and entorhinal cortices in the macaque monkey. J Neurosci 16:4757-4775.

Sobotka S, Ringo JL (1993) Investigation of long-term recognition and association memory in unit responses from inferotemporal cortex. Exp Brain Res 96:28-38.

Sobotka S, Ringo JL (1994) Stimulus specific adaptation in excited but not in inhibited cells in inferotemporal cortex of macaque. Brain Res 646:95-99.

Sobotka S, Ringo JL (1996) Mnemonic responses of single units recorded from monkey inferotemporal cortex, accessed via transcommissural versus direct pathways: a dissociation between unit activity and behavior. J Neurosci 16:4222-4230.

Stark CEL, Squire LR (2000) Intact visual perceptual discrimination in humans in the absence of perirhinal cortex. Learn Mem 7:273-278.

Suzuki WA (1996) Neuroanatomy of the monkey entorhinal, perirhinal and parahippocampal cortices: organization of cortical inputs and interconnections with amygdala and striatum. Semin Neurosci 8:3-11.

Suzuki WA, Amaral DG (1994a) Perirhinal and parahippocampal cortices of the macaque monkey: cortical afferents. J Comp Neurol 350:497-533.

Suzuki WA, Amaral DG (1994b) Topographical organization of the reciprocal connections between monkey entorhinal cortex and the perirhinal and parahippocampal cortices. J Neurosci 14:1856-1877.

Tanaka K (1996) Inferotemporal cortex and object vision. Annu Rev Neurosci 19:109-139.

Tang Y, Mishkin M, Aigner TG (1997) Effects of muscarinic blockade in perirhinal cortex during visual recognition. Proc Natl Acad Sci USA 94:12667-12669.

Thornton JA, Rothblat LA, Murray EA (1997) Rhinal cortex removal produces amnesia for preoperatively learned discrimination problem but fails to disrupt postoperative acquisition and retention in rhesus monkeys. J Neurosci 17:8536-8549. 